\title{
DETERMINAÇÃO DE MINERAIS EM HORTALIÇAS ORGÂNICAS E CONVENCIONAIS CULTIVADAS NO VALE DO TAQUARI, RS
}

\section{DETERMINATION OF MINERALS IN ORGANIC AND CONVENTIONAL VEGETABLES GROWN IN VALE DO TAQUARI, RS}

\author{
Katiana Korndörfer ${ }^{1}$; Mônica Jachetti Maciel²; Claucia Fernanda Volken de Souza ${ }^{3}$ \\ ${ }^{1}$ Centro Universitário UNIVATES - Lajeado - Brasil kanaitak@ hotmail.com \\ ${ }^{2}$ Centro Universitário UNIVATES - Lajeado - Brasil moni.jm@ hotmail.com \\ ${ }^{3}$ Centro Universitário UNIVATES - Lajeado - Brasil claucia@ univates.br
}

\begin{abstract}
Resumo
O consumo de hortaliças tem sido estimulado pelos benefícios no combate às deficiências de vitaminas e sais minerais, contribuindo também, na prevenção de doenças crônicas não transmissíveis. Esses alimentos são importantes para uma dieta saudável, pois são fontes de alguns componentes funcionais. A agricultura orgânica não utiliza produto químico no processo produtivo, em contrapartida à agricultura convencional, cujo objetivo principal é o uso intensivo de terras e de tecnologias, emprega grande quantidade desses produtos. As hortaliças produzidas nas duas formas de cultivo possuem metais, necessários em pequenas quantidades na dieta dos seres vivos, porém níveis excessivos desses elementos podem ser extremamente tóxicos. O objetivo desse trabalho foi avaliar e comparar o teor de $\mathrm{Na}, \mathrm{K}, \mathrm{Ca}, \mathrm{Mg}, \mathrm{Cu}, \mathrm{Zn}, \mathrm{Fe}$ e $\mathrm{Mn}$ em amostras das porções comestíveis das hortaliças beterraba, cenoura, espinafre e repolho in natura de sistemas de cultivo orgânico e convencional produzidas no Vale do Taquari, RS. As análises foram executadas conforme metodologia oficial do Ministério da Agricultura, Pecuária e Abastecimento. O teor médio de sódio das hortaliças orgânicas analisadas foi maior que das convencionais. A hortaliça que apresentou maior quantidade de ferro foi o espinafre convencional, 5,18 $\mathrm{mg} / 100 \mathrm{~g}$. Somente houve diferença estatística significativa $(p<0,05)$ do cultivo orgânico em relação ao cultivo convencional para a hortaliça espinafre - para o mineral manganês - e para a hortaliça cenoura - para os minerais manganês e ferro. Nas condições deste estudo as culturas orgânicas de beterraba, cenoura, espinafre e repolho não apresentaram qualidade nutricional superior em relação às convencionais.
\end{abstract}

Palavras-chave: minerais; hortaliças orgânicas; hortaliças convencionais.

\section{Introdução}

A alimentação fornece aos seres humanos diversos elementos minerais (WELCH e GRAHAM, 2004; GRUSAK e CAKMAK, 2005). O consumo de vegetais - principal fonte de minerais na dieta humana (MARTÍNEZ-BALLESTA et al., 2010) - está associado com a redução 
de doenças cardiovasculares, neurológicas e inúmeros tipos de câncer, além da prevenção de diversas enfermidades (ABEROUMAND e DEOKULE, 2009).

A beterraba - uma das hortaliças mais ricas em $\mathrm{Fe}$ - é também fonte de $\mathrm{K}, \mathrm{Na}, \mathrm{Cu}$ e $\mathrm{Zn}$ (KANNER et al., 2001; HORTA et al., 2004). A cenoura apresenta elementos como Ca, Fe, Na e Zn, além de elevado conteúdo de K (EMBRAPA, 2013). O espinafre é um dos vegetais folhosos mais consumidos em todo o mundo (NISHA et al., 2004) e apresenta considerável teor de Fe e de Ca (NISHIRA et al., 2001; SILVA, 2006). O repolho é a quinta hortaliça mais produzida no Brasil, uma das razões é a excelente composição nutricional (SILVA JUNIOR, 1991), tendo em vista que contém elevado teor de sais minerais indispensáveis para a dieta humana (FONTANÉTTI et al., 2006).

Os alimentos orgânicos despertam o interesse dos consumidores, pois nesse tipo de agricultura não é permitido o uso de substâncias químicas que coloquem em risco a saúde humana e o meio ambiente, tais como fertilizantes sintéticos e agrotóxicos, e de sementes transgênicas. O vegetal orgânico é produzido num ambiente onde se contempla o uso responsável do solo, da água, do ar e dos demais recursos naturais, respeitando as relações sociais e culturais (BRASIL, 2013). Muitos consumidores acreditam que os alimentos orgânicos sejam mais nutritivos e saudáveis que os convencionais, no entanto, os possíveis efeitos destes produtos orgânicos sobre a saúde ainda não foram cientificamente documentados, sendo necessários mais estudos (MAGKOS et al., 2003; WORTHINGTON, 2001). Estima-se que no Brasil, em 2011, o setor de produtos orgânicos tenha movimentado cerca de 500 milhões de reais e espera-se para 2014 um crescimento que ultrapasse os 46\% (ASSOCIAÇÃO BRASILEIRA DE ORGÂNICOS, 2013).

O cultivo convencional, predominante no Brasil, cresceu muito com a modernização da agricultura proporcionando, além de novas técnicas e equipamentos, uma diversidade de insumos, como agrotóxicos e fertilizantes (STOPPELLI, 2005). Este cultivo utiliza as terras e a tecnologia, envolvendo mecanização e elevado uso de insumos como fertilizantes, herbicidas e inseticidas, ocasionando, em curto prazo, resultados econômicos expressivos (MERTEN e MINELLA, 2002). Entretanto, este tipo de cultivo é questionado, pois os agrotóxicos fazem mal à saúde do consumidor, além de contaminarem o meio ambiente (ARCANJO et al., 2001).

Portanto, esse trabalho teve como objetivo avaliar e comparar o teor de $\mathrm{Na}, \mathrm{K}, \mathrm{Ca}, \mathrm{Mg}, \mathrm{Cu}$, $\mathrm{Zn}, \mathrm{Fe}$ e Mn em amostras das porções comestíveis das hortaliças: beterraba, cenoura, espinafre e repolho in natura de sistemas de cultivo orgânico e convencional produzidas no Vale do Taquari, RS.

\section{Material e métodos}


As amostras de hortaliças cultivadas sob o sistema orgânico foram coletadas em uma propriedade de hortigranjeiros, localizada no município de Cruzeiro do Sul, na região do Vale do Taquari/RS, certificada pela ECOCERT Brasil (Florianópolis, SC) como produtora de hortaliças orgânicas, sob o registro 2853BR. As amostras de cultivo convencional foram obtidas em outra propriedade do mesmo município junto a um produtor de hortaliças de cultivo convencional.

As hortaliças escolhidas para análise foram: beterraba (Beta vulgaris L. var catrine), cenoura (Daucus carota L. var brasilia), espinafre (Spinacea oleracea L.) e repolho (Brassica oleracea L. var capitata). Foram coletadas nove amostras (três coletas mensais) de cada vegetal e de cada sistema de produção (orgânico e convencional) no período de julho a setembro. As amostras foram coletadas logo após a colheita pelo produtor, como plantas inteiras, com separação posterior das partes comestíveis. Estas foram higienizadas em água potável e acondicionadas em sacos plásticos fechados e armazenadas em refrigerador por um dia. As amostras de cenoura e beterraba foram processadas em ralador manual, enquanto que as amostras de espinafre e repolho foram picadas manualmente.

A partir do material resultante da análise de cinzas (IAL, 2008), os nutrientes minerais analisados foram: $\mathrm{Na}, \mathrm{K}, \mathrm{Ca}, \mathrm{Mg}, \mathrm{Cu}, \mathrm{Zn}, \mathrm{Fe}$ e $\mathrm{Mn}$. As cinzas foram submetidas à digestão ácida com ácido clorídrico 1:1, filtradas em papel de filtro qualitativo e diluídas. A quantificação destes minerais foi feita por Espectrofotometria de Absorção Atômica com Chama (EAA) em equipamento segundo metodologia oficial do Ministério da Agricultura, Pecuária e Abastecimento (BRASIL, 1991).

O delineamento experimental foi o inteiramente casualizado com nove repetições em triplicata. Os resultados das determinações analíticas para beterraba, cenoura, espinafre e repolho de ambos os sistemas de cultivo foram comparados através do teste $\mathrm{t}(\mathrm{p}<0,05)$, empregando o software Statistica® for Windows versão 7.0.

\section{Resultados e discussão}

A Tabela 1 apresenta os resultados das análises de minerais (mg/100 g de amostra) da beterraba convencional e orgânica. Com exceção do $\mathrm{K}$, os valores médios dos minerais nas amostras de beterraba foram maiores no cultivo orgânico do que no convencional, no entanto, nenhum dos minerais avaliados apresentou diferença significativa entre os dois sistemas de cultivo. Silva (2011), ao verificar a quantidade de ferro em beterrabas orgânicas e convencionais também não verificou diferença significativa entre os cultivos. 
Tabela 1- Resultados dos minerais (mg/100 g de amostra) da beterraba convencional e orgânica

\begin{tabular}{|c|c|c|c|c|c|c|c|c|c|c|c|c|c|c|c|c|}
\hline \multirow[b]{2}{*}{ Mês } & \multicolumn{2}{|c|}{$\mathrm{Na}$} & \multicolumn{2}{|c|}{$\mathbf{K}$} & \multicolumn{2}{|c|}{$\mathrm{Ca}$} & \multicolumn{2}{|c|}{$\mathrm{Mg}$} & \multicolumn{2}{|c|}{$\mathrm{Cu}$} & \multicolumn{2}{|c|}{ Zn } & \multicolumn{2}{|c|}{$\mathbf{F e}$} & \multicolumn{2}{|c|}{ Mn } \\
\hline & C & $\mathbf{O}$ & C & $\mathbf{O}$ & C & $\mathbf{O}$ & C & $\mathbf{O}$ & $\mathrm{C}$ & $\mathbf{O}$ & $\mathrm{C}$ & $\mathbf{O}$ & C & $\mathbf{O}$ & $\mathrm{C}$ & $\mathbf{O}$ \\
\hline \multirow{3}{*}{$\mathbf{J}$} & 45,52 & 91,40 & 207,14 & 90,71 & 22,29 & 27,05 & 20,68 & 22,83 & 0,00 & 0,15 & 0,51 & 0,73 & 0,59 & 2,58 & 0,25 & 0,55 \\
\hline & 27,49 & 14,31 & 234,33 & 122,20 & 20,65 & 17,05 & 25,39 & 18,70 & 0,00 & 0,01 & 0,96 & 0,81 & 0,42 & 0,36 & 0,40 & 0,21 \\
\hline & - & 44,35 & - & 116,45 & - & 21,93 & - & 51,49 & - & 0,06 & - & 1,67 & - & 4,09 & - & 0,50 \\
\hline \multirow{3}{*}{$\mathbf{A}$} & 62,01 & 40,00 & 226,19 & 143,35 & 2,17 & 1,37 & 2,29 & 2,50 & 0,17 & 0,35 & 0,01 & 0,01 & 0,69 & 1,28 & 0,12 & 0,44 \\
\hline & 17,44 & 27,72 & 206,45 & 147,26 & 2,44 & 1,40 & 3,34 & 3,31 & 0,27 & 0,27 & 0,72 & 0,45 & 0,97 & 1,14 & 0,50 & 0,30 \\
\hline & 16,09 & 21,31 & 123,33 & 118,29 & 1,64 & 1,65 & 2,35 & 2,62 & 0,18 & 0,14 & 0,34 & 0,53 & 2,03 & 1,03 & 0,37 & 0,31 \\
\hline \multirow{3}{*}{$\mathbf{S}$} & 12,92 & 31,12 & 142,70 & 103,04 & 27,13 & 10,39 & 23,80 & 20,80 & 0,16 & 0,18 & 0,71 & 0,38 & 0,58 & 0,71 & 0,91 & 1,17 \\
\hline & 20,58 & 95,15 & 76,41 & 127,43 & 10,98 & 18,74 & 5,02 & 24,04 & 0,10 & 0,09 & 0,56 & 0,44 & 0,22 & 0,64 & 0,06 & 0,35 \\
\hline & 15,57 & 111,25 & 88,78 & 152,82 & 19,05 & 21,94 & 23,07 & 24,04 & 0,27 & 0,18 & 0,63 & 0,61 & 0,53 & 1,34 & 0,40 & 0,54 \\
\hline Média & $27,20^{\mathrm{a}}$ & $52,96^{\mathrm{a}}$ & $163,17^{\mathrm{a}}$ & $124,62^{\mathrm{a}}$ & $13,30^{\mathrm{a}}$ & $13,50^{\mathrm{a}}$ & $13,24^{a}$ & $18,93^{\mathrm{a}}$ & $0,14^{\mathrm{a}}$ & $0,16^{\mathrm{a}}$ & $0,55^{\mathrm{a}}$ & $0,63^{\mathrm{a}}$ & $0,75^{\mathrm{a}}$ & $1,46^{\mathrm{a}}$ & $0,38^{a}$ & $0,49^{\mathrm{a}}$ \\
\hline Desvio & 17,52 & 36,24 & 63,14 & 20,60 & 10,30 & 10,06 & 10,79 & 15,46 & 0,11 & 0,10 & 0,29 & 0,45 & 0,56 & 1,17 & 0,26 & 0,28 \\
\hline
\end{tabular}

Na Tabela 2 são apresentados os resultados das análises dos minerais (mg/100 g de amostra) da cenoura convencional e orgânica. Para a cenoura as médias de $\mathrm{K}, \mathrm{Cu}, \mathrm{Zn}$ e $\mathrm{Mn}$ foram maiores no sistema de cultivo convencional e $\mathrm{Na}, \mathrm{Ca}, \mathrm{Mg}$ e Fe para o sistema de cultivo orgânico. Dos minerais avaliados, apenas o Mn e o Fe apresentaram diferença significativa $(\mathrm{p}<0,05)$ entre os dois sistemas de cultivo. O valor médio do teor de Mn do cultivo convencional foi $100 \%$ maior que do cultivo orgânico e o Fe apresentou média cerca de $80 \%$ superior no cultivo orgânico em relação ao convencional.

Tabela 2- Resultados das análises dos minerais (mg/100 g de amostra) da cenoura convencional e orgânica

\begin{tabular}{|c|c|c|c|c|c|c|c|c|c|c|c|c|c|c|c|c|}
\hline \multirow[b]{2}{*}{ Mês } & \multicolumn{2}{|c|}{$\mathrm{Na}$} & \multicolumn{2}{|c|}{$\mathbf{K}$} & \multicolumn{2}{|c|}{$\mathrm{Ca}$} & \multicolumn{2}{|c|}{ Mg } & \multicolumn{2}{|c|}{$\mathbf{C u}$} & \multicolumn{2}{|c|}{$\mathbf{Z n}$} & \multicolumn{2}{|c|}{$\mathbf{F e}$} & \multicolumn{2}{|c|}{ Mn } \\
\hline & C & O & $\mathbf{C}$ & O & C & O & C & $\mathbf{O}$ & $\mathrm{C}$ & O & $\mathrm{C}$ & $\mathbf{O}$ & C & $\mathbf{O}$ & $\mathrm{C}$ & O \\
\hline \multirow{3}{*}{$\mathbf{J}$} & 36,04 & 39,73 & 133,72 & 110,06 & 32,76 & 31,46 & 10,81 & 16,43 & 0,05 & 0,02 & 0,21 & 0,27 & 0,87 & 0,70 & 0,10 & 0,14 \\
\hline & 43,67 & 47,61 & 96,50 & 99,36 & 28,49 & 40,51 & 14,68 & 13,04 & 0,03 & 0,08 & 0,23 & 0,14 & 0,87 & 2,70 & 0,15 & 0,14 \\
\hline & 38,94 & 42,58 & 54,75 & 113,25 & 32,08 & 37,38 & 15,84 & 10,67 & 0,01 & 0,03 & 1,45 & 0,21 & 1,90 & 1,39 & 0,22 & 0,09 \\
\hline \multirow{3}{*}{ A } & 33,59 & 15,30 & 441,50 & 139,39 & 2,08 & 2,56 & 1,23 & 1,11 & 0,15 & 0,10 & 0,28 & 0,22 & 0,77 & 0,48 & 0,23 & 0,08 \\
\hline & 8,08 & 19,84 & 174,48 & 115,88 & 2,61 & 2,98 & 1,74 & 1,41 & 0,11 & 0,11 & 0,29 & 0,15 & 1,15 & 1,37 & 0,17 & 0,10 \\
\hline & 6,88 & 22,40 & 52,89 & 148,55 & 1,97 & 2,25 & 1,25 & 1,22 & 0,13 & 0,10 & 0,31 & 0,32 & 0,43 & 0,85 & 0,22 & 0,12 \\
\hline \multirow{3}{*}{$\mathbf{S}$} & 16,38 & 15,86 & 145,78 & 128,79 & 20,58 & 39,30 & 6,80 & 16,97 & 0,12 & 0,08 & 0,22 & 0,15 & 0,29 & 2,15 & 0,14 & 0,07 \\
\hline & 20,41 & 81,47 & 192,62 & 11,49 & 33,94 & 41,29 & 13,41 & 11,49 & 0,13 & 0,05 & 0,88 & 0,32 & 0,49 & 1,36 & 0,29 & 0,08 \\
\hline & 30,70 & 79,95 & 101,55 & 11,05 & 25,60 & 36,91 & 7,90 & 11,05 & 0,11 & 0,08 & 0,31 & 0,28 & 0,27 & 1,60 & 0,30 & 0,09 \\
\hline Média & $26,08^{a}$ & $40,53^{a}$ & $154,87^{\mathrm{a}}$ & $97,54^{\mathrm{a}}$ & $20,01^{\mathrm{a}}$ & $26,07^{\mathrm{a}}$ & $8,19^{\mathrm{a}}$ & $9,26^{\mathrm{a}}$ & $0,09^{\mathrm{a}}$ & $0,07^{\mathrm{a}}$ & $0,46^{\mathrm{a}}$ & $0,23^{\mathrm{a}}$ & $0,78^{\mathrm{b}}$ & $1,40^{\mathrm{a}}$ & $0,20^{\mathrm{a}}$ & $0,10^{\mathrm{b}}$ \\
\hline Desvio & 13,56 & 25,71 & 117,88 & 51,19 & 13,94 & 17,83 & 5,86 & 6,40 & 0,05 & 0,03 & 0,42 & 0,07 & 0,51 & 0,70 & 0,07 & 0,03 \\
\hline
\end{tabular}


A literatura apresenta poucos estudos recentes sobre o conteúdo de minerais em hortaliças orgânicas e convencionais. No trabalho de Worthington (2001), a cenoura orgânica apresentou 12\% a mais de ferro do que a convencional.

Na Tabela 3 são apresentados os resultados das análises dos minerais (mg/100 g de amostra) do espinafre convencional e orgânico. Os teores médios dos seguintes minerais para o espinafre foram superiores no cultivo convencional: $\mathrm{K}, \mathrm{Ca}, \mathrm{Mg}$ e $\mathrm{Fe}$, enquanto que no cultivo orgânico foram maiores os teores de $\mathrm{Na}, \mathrm{Cu}, \mathrm{Zn}$ e $\mathrm{Mn}$, mas somente o $\mathrm{Mn}$ apresentou diferença significativa ( $\mathrm{p}<$ $0,05)$ entre os dois sistemas de cultivo. O Fe apresentou uma média cerca de $130 \%$ maior no sistema convencional em relação ao orgânico. Os teores de $\mathrm{Na}, \mathrm{Ca}, \mathrm{Mg}, \mathrm{Fe}$ e $\mathrm{Mn}$ do espinafre foram superiores às demais hortaliças avaliadas no presente estudo.

Tabela 3- Resultados das análises dos minerais (mg/100 g de amostra) do espinafre convencional e orgânico

\begin{tabular}{|c|c|c|c|c|c|c|c|c|c|c|c|c|c|c|c|c|}
\hline \multirow[b]{2}{*}{ Mês } & \multicolumn{2}{|c|}{$\mathrm{Na}$} & \multicolumn{2}{|c|}{$\mathbf{K}$} & \multicolumn{2}{|c|}{ Ca } & \multicolumn{2}{|c|}{ Mg } & \multicolumn{2}{|c|}{$\mathrm{Cu}$} & \multicolumn{2}{|c|}{ Zn } & \multicolumn{2}{|c|}{$\mathrm{Fe}$} & \multicolumn{2}{|c|}{ Mn } \\
\hline & C & 0 & $\mathrm{C}$ & $\mathbf{O}$ & C & $\mathbf{O}$ & $\mathrm{C}$ & $\mathbf{O}$ & $\mathrm{C}$ & O & $\mathrm{C}$ & O & $\mathrm{C}$ & $\mathbf{O}$ & $\mathrm{C}$ & $\mathbf{O}$ \\
\hline \multirow{3}{*}{$\mathbf{J}$} & 110,15 & 114,20 & 110,69 & 114,08 & 147,61 & 134,60 & 68,87 & 99,92 & 0,00 & 0,10 & 0,36 & 0,76 & 7,00 & 3,27 & 1,26 & 5,15 \\
\hline & 100,24 & 94,17 & 115,95 & 94,28 & 162,15 & 73,42 & 150,0 & 43,42 & 0,04 & 0,18 & 0,56 & 0,57 & 6,34 & 2,07 & 1,69 & 8,42 \\
\hline & 122,02 & 86,94 & 101,90 & 104,79 & 159,79 & 62,46 & 128,2 & 38,58 & 0,00 & 0,18 & 0,50 & 0,36 & 8,85 & 3,45 & 2,61 & 4,10 \\
\hline \multirow{3}{*}{$\mathbf{A}$} & 68,93 & 127,29 & 330,83 & 186,89 & 6,45 & 9,53 & 3,99 & 5,37 & 0,21 & 0,16 & 0,04 & 0,04 & 3,40 & 2,40 & 0,93 & 4,63 \\
\hline & 84,92 & 142,83 & 205,84 & 80,86 & 7,57 & 8,31 & 6,10 & 4,52 & 0,28 & 0,24 & 0,40 & 0,44 & 15,75 & 3,49 & 0,96 & 3,39 \\
\hline & 37,22 & 188,08 & 170,35 & 115,57 & 5,59 & 14,27 & 4,71 & 5,58 & 0,20 & 0,14 & 0,29 & 0,35 & 1,60 & 2,39 & 0,60 & 3,21 \\
\hline \multirow{3}{*}{$\mathbf{S}$} & 102,24 & 126,74 & 207,92 & 143,99 & 33,78 & 41,97 & 32,93 & 25,06 & 0,10 & 0,24 & 0,46 & 0,45 & 1,98 & 1,43 & 0,62 & 7,45 \\
\hline & 91,83 & 176,63 & 244,14 & 148,50 & 62,44 & 45,57 & 28,71 & 27,59 & 0,08 & 0,13 & 0,26 & 0,66 & 0,62 & 0,95 & 0,69 & 5,58 \\
\hline & 184,62 & 186,04 & 86,65 & 196,95 & 65,56 & 62,34 & 34,35 & 37,10 & 0,22 & 0,14 & 0,56 & 0,43 & 1,12 & 1,08 & 0,78 & 3,09 \\
\hline Média & $100,2^{\mathrm{a}}$ & $138,1^{\mathrm{a}}$ & $174,9^{\mathrm{a}}$ & $131,77^{\mathrm{a}}$ & $72,33^{\mathrm{a}}$ & $50,27^{\mathrm{a}}$ & $50,88^{\mathrm{a}}$ & $31,90^{\mathrm{a}}$ & $0,13^{\mathrm{a}}$ & $0,17^{\mathrm{a}}$ & $0,38^{\mathrm{a}}$ & $0,45^{\mathrm{a}}$ & $5,18^{\mathrm{a}}$ & $2,28^{\mathrm{a}}$ & $1,13^{\mathrm{b}}$ & $5,00^{\mathrm{a}}$ \\
\hline Desvio & 40,24 & 38,19 & 80,54 & 40,34 & 67,09 & 39,92 & 54,27 & 29,67 & 0,11 & 0,05 & 0,17 & 0,21 & 4,92 & 0,99 & 0,66 & 1,89 \\
\hline
\end{tabular}

Rembialkowska (2000) encontrou quantidades superiores de ferro, potássio e cálcio na cenoura e no espinafre orgânico, quando relacionados ao cultivo convencional. A cenoura orgânica também apresentou quantidade superior de magnésio. Neste estudo, o cultivo convencional apresentou valores maiores para esses minerais e hortaliças, com exceção do ferro, cálcio e magnésio da cenoura orgânica. No estudo de Worthington (2001), o espinafre orgânico apresentou $25 \%$ a mais de ferro e $13 \%$ a menos de magnésio, frente ao convencional. Comparando-se aos valores encontrados, o espinafre convencional apresentou quantidade maior de ferro (127\%) e o espinafre orgânico apresentou quantidade cerca de 59\% superior de magnésio.

A Tabela 4 apresenta os resultados dos minerais $(\mathrm{mg} / 100 \mathrm{~g})$ do repolho convencional e orgânico. As médias dos teores de $\mathrm{Cu}, \mathrm{Zn}$ e $\mathrm{Mn}$ do repolho foram superiores no cultivo convencional, enquanto que no cultivo orgânico as concentrações de $\mathrm{Na}, \mathrm{K}, \mathrm{Ca}, \mathrm{Mg}$ e Fe foram 
maiores, contudo, nenhum dos minerais avaliados apresentou diferença significativa $(\mathrm{p}<0,05)$ entre os dois sistemas de cultivos. No estudo de Worthington (2001) o repolho orgânico apresentou $41 \%$ de ferro e $40 \%$ de magnésio em quantidades superiores ao convencional, porém no presente trabalho os valores foram menores, 8,5 e $1,7 \%$, respectivamente.

Tabela 4- Resultados das análises dos minerais (mg/100 g de amostra) de repolho convencional e orgânico

\begin{tabular}{|c|c|c|c|c|c|c|c|c|c|c|c|c|c|c|c|c|}
\hline \multirow[b]{2}{*}{ Mês } & \multicolumn{2}{|c|}{$\mathrm{Na}$} & \multicolumn{2}{|c|}{$\mathbf{K}$} & \multicolumn{2}{|c|}{$\mathrm{Ca}$} & \multicolumn{2}{|c|}{ Mg } & \multicolumn{2}{|c|}{$\mathrm{Cu}$} & \multicolumn{2}{|c|}{$\mathrm{Zn}$} & \multicolumn{2}{|c|}{$\mathrm{Fe}$} & \multicolumn{2}{|c|}{ Mn } \\
\hline & C & $\mathbf{O}$ & C & $\mathbf{O}$ & C & 0 & $\mathrm{C}$ & O & C & $\mathbf{O}$ & $\mathrm{C}$ & O & $\mathbf{C}$ & $\mathbf{O}$ & $\mathbf{C}$ & O \\
\hline \multirow{4}{*}{$\mathbf{J}$} & 13,18 & 9,32 & 145,60 & 113,06 & 57,17 & 70,38 & 16,10 & 19,13 & 0,00 & 0,08 & 0,31 & 0,31 & 0,38 & 0,37 & 0,19 & 0,33 \\
\hline & 4,03 & 22,48 & 128,11 & 243,93 & 35,03 & 73,33 & 10,74 & 13,67 & 0,00 & 0,01 & 0,44 & 0,44 & 0,21 & 0,43 & 0,37 & 0,17 \\
\hline & 11,96 & 12,40 & 135,83 & 82,37 & 51,29 & 50,48 & 14,80 & 14,43 & 0,01 & 0,00 & 0,61 & 0,31 & 0,30 & 0,45 & 0,28 & 0,12 \\
\hline & 5,51 & 24,73 & 107,27 & 121,55 & 2,40 & 3,10 & 1,44 & 1,02 & 0,16 & 0,20 & 0,20 & 0,52 & 0,48 & 0,60 & 0,13 & 0,15 \\
\hline \multirow[t]{3}{*}{ A } & 3,02 & 7,80 & 128,11 & 31,74 & 3,30 & 2,88 & 1,54 & 1,14 & 0,18 & 0,04 & 0,27 & 0,14 & 0,39 & 0,24 & 0,16 & 0,10 \\
\hline & 5,27 & 7,03 & 65,41 & 95,51 & 2,48 & 3,11 & 1,45 & 1,13 & 0,14 & 0,06 & 0,31 & 0,17 & 0,43 & 0,51 & 0,22 & 0,13 \\
\hline & 7,09 & 11,62 & 120,17 & 130,23 & 38,70 & 49,28 & 9,73 & 17,07 & 0,11 & 0,06 & 0,26 & 0,24 & 0,31 & 0,29 & 0,28 & 0,12 \\
\hline \multirow[t]{2}{*}{$\mathbf{S}$} & 8,37 & 5,71 & 100,97 & 111,25 & 55,53 & 41,64 & 17,64 & 11,63 & 0,07 & 0,03 & 0,25 & 0,29 & 0,30 & 0,26 & 0,12 & 0,10 \\
\hline & 9,10 & 5,93 & 38,57 & 104,24 & 50,72 & 52,90 & 15,78 & 11,50 & 0,14 & 0,04 & 0,36 & 0,27 & 0,38 & 0,25 & 0,13 & 0,11 \\
\hline Média & $7,50^{\mathrm{a}}$ & $11,89^{\mathrm{a}}$ & $107,78^{a}$ & $114,88^{\mathrm{a}}$ & $32,96^{\mathrm{a}}$ & $38,57^{\mathrm{a}}$ & $9,91^{\mathrm{a}}$ & $10,08^{\mathrm{a}}$ & $0,09^{\mathrm{a}}$ & $0,06^{\mathrm{a}}$ & $0,33^{\mathrm{a}}$ & $0,30^{\mathrm{a}}$ & $0,35^{\mathrm{a}}$ & $0,38^{\mathrm{a}}$ & $0,21^{\mathrm{a}}$ & $0,15^{\mathrm{a}}$ \\
\hline Desvio & 3,48 & 7,05 & 35,05 & 56,36 & 23,79 & 28,46 & 6,81 & 7,15 & 0,07 & 0,06 & 0,12 & 0,12 & 0,08 & 0,13 & 0,09 & 0,07 \\
\hline
\end{tabular}

Masamba e Nguyen (2008) no repolho e na cenoura orgânica encontraram maiores valores de cálcio, de 12 a 16\%, enquanto que o potássio, 2 a 17\%. No presente trabalho o repolho orgânico apresentou valores superiores para o cálcio $(+17 \%)$ e para o potássio $(+6,5 \%)$.

Schuphan (1974) mostrou que espinafre, cenoura e repolho provenientes de cultivo orgânico continham mais ferro $(+77 \%)$, potássio $(+18 \%)$ e cálcio $(+10 \%)$ e um teor mais baixo de sódio ($12 \%$ ) em comparação aos vegetais do sistema de cultivo convencional. Ao relacionar com os dados obtidos no presente trabalho, a cenoura orgânica apresentou valores maiores para o ferro (+79\%), cálcio (+30\%) e sódio (+55\%), o repolho orgânico apresentou valores maiores para o ferro $(+8,5)$, potássio (+6,5\%), cálcio $(+17 \%)$ e sódio $(+58 \%)$ em relação ao cultivo convencional. Já o espinafre orgânico apresentou valores de sódio $(+37 \%)$. Nos demais minerais nas hortaliças mencionadas previamente, o valor do cultivo convencional foi maior do que o orgânico.

Dangour et al. (2009) constataram que não havia diferença significativa entre as quantidades dos minerais magnésio, cálcio, potássio, zinco e cobre, ao comparar diversos tipos de alimentos, dentre eles, vegetais e frutas, em relação ao modo de cultivo.

Os resultados encontrados demonstraram que os vegetais orgânicos possuem uma maior quantidade de $\mathrm{Na}$ em relação aos cultivados de forma convencional, chegando a uma diferença de quase $95 \%$ em relação à beterraba, aproximadamente $55 \%$ na cenoura e no repolho e cerca de $38 \%$ no espinafre. 
Segundo IBGE (2013), o teor de potássio da cenoura é de $230 \mathrm{mg} / 100$ g. Nesse estudo, os valores médios dessa hortaliça, no modo orgânico e convencional, foram 135 e 48\% maiores, respectivamente. A beterraba, para esse mesmo mineral, apresentou um valor cerca de 2,5 vezes maior do que a hortaliça convencional e o espinafre, aproximadamente 3,5 vezes maior do que nesse trabalho. No repolho o valor de potássio encontrado (IBGE, 2013) foi de $170 \mathrm{mg} / 100 \mathrm{~g}, 57 \%$ maior do que o de cultivo convencional desse estudo.

O magnésio está, principalmente, em vegetais verde-escuros (JARDIM et al., 2004), no entanto, a hortaliça que apresentou o maior teor desse mineral foi o repolho cultivado de forma convencional $(50,88 \mathrm{mg} / 100 \mathrm{~g})$. Já a cenoura convencional apresentou a menor quantidade de magnésio $(8,19 \mathrm{mg} / 100 \mathrm{~g})$.

O zinco é necessário em pequenas quantidades no organismo, por isso é denominado de micronutriente, sendo de $7 \mathrm{mg}$ a ingestão diária recomendada para adultos (ANVISA, 2005). A maior concentração de zinco foi encontrada na beterraba orgânica $(0,63 \mathrm{mg} / 100 \mathrm{~g})$ e a menor na cenoura orgânica $(0,23 \mathrm{mg} / 100 \mathrm{~g})$.

A hortaliça que apresentou uma maior quantidade de ferro foi o espinafre cultivado de modo convencional, 5,18 mg/100 g. O espinafre teve um valor estabelecido pelo IBGE (2013) de 3,57 mg de Fe/100 g. A quantidade de Fe da cenoura e da beterraba é de 0,72 mg e 0,80 mg/100 g, respectivamente (IBGE, 2013). Resultados semelhantes foram obtidos no presente trabalho para essas hortaliças cultivadas de forma convencional, sendo $0,78 \mathrm{mg} / 100 \mathrm{~g}$ para a cenoura e 0,75 $\mathrm{mg} / 100 \mathrm{~g}$ para a beterraba. Para as mesmas hortaliças, cultivadas em sistema orgânico, os teores praticamente dobraram, sendo $1,40 \mathrm{mg} / 100 \mathrm{~g}$ para a cenoura e $1,46 \mathrm{mg} / 100 \mathrm{~g}$ para a beterraba.

As hortaliças apresentaram quantidades distintas em relação aos minerais e também ao tipo de cultivo. Isso pode ter ocorrido devido a composição química dos vegetais que podem variar e apresentar diferenças em função de fatores associados ao cultivo e ao ambiente, como local de plantio, adubação, ocorrência de pragas, diferenças edafoclimáticas, período de colheita, idade e características genéticas da planta, entre outros (SMITH, 1993).

\section{Conclusão}

Como pode ser observado não houve uma homogeneidade nas médias encontradas nas hortaliças para os minerais quanto à forma de cultivo. Somente houve diferença estatística significativa do cultivo orgânico em relação ao cultivo convencional para a hortaliça espinafre para o mineral manganês - e para a hortaliça cenoura - para os minerais manganês e ferro. Nas condições deste estudo as culturas orgânicas de beterraba, cenoura, espinafre e repolho não apresentaram qualidade nutricional superior em relação às convencionais. 


\begin{abstract}
The consumption of vegetables has been stimulated by the benefits to combat deficiencies of vitamins and minerals, contributing also in the prevention of chronic diseases. These foods are important for a healthy diet, as a source of functional components. Organic farming does not use chemicals in the production process, in contrast to conventional farming whose main objective is the intensive use of land and technology, employs large amounts of these products. The vegetables produced in two forms of cultivation have metals, needed in small amounts in the diet of living beings, but excessive levels of these elements can be extremely toxic. The aim of this work was to evaluate and compare the content of $\mathrm{Na}, \mathrm{K}, \mathrm{Ca}, \mathrm{Mg}, \mathrm{Cu}, \mathrm{Zn}, \mathrm{Fe}$ and $\mathrm{Mn}$ in samples of edible portions of vegetables beets, carrots, spinach and cabbage fresh from organic farming systems and conventional Vale do Taquari, RS. The analyzes were performed according to the official methodology of the Ministry of Agriculture, Livestock and Supply. The average content of sodium organic vegetables analyzed was higher than the conventional. The vegetable with the highest amount of iron was the spinach conventional, $5.18 \mathrm{mg} / 100 \mathrm{~g}$. Only statistically significant ( $p<$ $0,05)$ differences of organic farming compared to conventional tillage for vegetable spinach - for the mineral manganese - and for vegetable carrot - for the minerals manganese and iron. In this study the cultures of organic beets, carrots, cabbage and spinach showed no superior nutritional quality compared to conventional.
\end{abstract}

Key-words: minerals, organic vegetables, conventional vegetables.

\title{
Referências
}

ABEROumAnD, A.; DEOKUlE, S.S. Determination of Elements Profile of Some Wild Edible Plants. Food Analytical Method, v. 2, p. 116-119, 2009.

AGÊNCIA NACIONAL DE VIGILÂNCIA SANITÁRIA (ANVISA). Resolução RDC n. ${ }^{\circ}$ 269, de 22 de setembro de 2005. Aprova o "Regulamento técnico sobre a ingestão diária recomendada (IDR) de proteína, vitaminas e minerais".

ARCANJO, L.R.; BRITO, K.F.W.; SAUERBECK, S. Alimentos orgânicos em Curitiba: consumo e significado. Contemporary Argumentation \& Debate, v. 8, p. 1-6, 2001.

ASSOCIAÇÃO BRASILEIRA DE ORGÂNICOS (BrasilBio). Disponível em: <http://www.brasilbio.com.br/en/>. Acesso em: 24 jan. 2013.

BRASIL, Ministério da Agricultura, Pecuária e Abastecimento, Secretaria de Defesa Agropecuária. Portaria no ${ }^{\text {108, de }}$ 04 de setembro de 1991. Métodos analíticos para controle de alimentos de alimentos para uso animal. Diário Oficial da União, Brasília, DF, 17 de setembro 1991. Seção 1, p. 19835.

BRASIL, Ministério da Agricultura, Pecuária e do Abastecimento. O que são produtos orgânicos, disponível em: $<$ http://www.prefiraorganicos.com.br/oquesao.aspx>, acesso em 01 fev. 2013.

DANGOUR, A.D. et al. Nutritional quality of organic foods: a systematic review. American Journal of Clinical Nutrition, v. 90, p. 680-5, 2009.

EMBRAPA. Comércio internacional de produtos hortícolas, leguminosas, raízes e tubérculos. Disponível em: http://www.cnph.embrapa.br/paginas/hortalicas_em_numeros/Gráfico\%20Balança\%20Comecial\%20de\%20Produtos\% 20Hortícolas,\%202009-1997.xls. Acesso em 23 jan. 2013.

FOnTANÉTTI, A.; CARVAlHO, G.J.; GOMES, L.A.A.; ALMEIDA, K.; MORAES, S.R.G.; TEIXEIRA, C.M. Adubação verde na produção orgânica de alface americana e repolho. Horticultura Brasileira, v. 24, p. 146-150, 2006. 
GRUSAK, M.A.; CAKMAK I. Methods to improve the crop delivery of minerals to human and livestock. In: BROADLEY, M.R.; WHITE, P.J. (Eds.). Plant Nutritional Genomics. Blackwel, p. 265-286, 2005.

HORTA, A.C.S.; SANTOS, H.S.; CONSTANTIN, J.; SCAPIM, C.A. Interferência de plantas daninhas na beterraba transplantada e semeada diretamente. Acta Scientiarum Agronomy, v.26, p.47-53, 2004.

IAL. Instituto Adolfo Lutz. Métodos físico-químicos para análise de alimentos. São Paulo: Instituto Adolfo Lutz, 4.ed, 2008.

IBGE- Instituto Brasileiro de Geografia e Estatística- Tabela de composição nutricional dos alimentos consumidos no Brasil 2008-2009. Disponível /visualizacao/monografias/GEBIS\%20\%20RJ/pof/Tabelas\%20de\%20composicao\%20nutricional\%20dos\%20alimentos \%20consumidos\%20no\%20Brasil_2008_2009.pdf>. Acesso em 05 jan. 2013.

JARDIM, P.C.B.V.; MONEGO, E.T.; REIS, M.A.C. Potássio, cálcio, magnésio e hipertensão arterial / Potassium, calcium, magnesium and hypertension. Revista Brasileira de Hipertensão, v. 11, n. 2, p. 109-111, 2004.

KANNER, J.; HAREL, S.; GRANIT, R. Betalains: A new class of dietary cationized antioxidants. Journal of Agricultural and Food Chemistry, v.49, p.5178-5185, 2001.

MAGKOS, F.; ARVANITI, F.; ZAMPELAS, A. Putting the safety of organic food into perspective. Nutrition Research Reviews, v. 16, p. 211-222, 2003.

MARTÍNEZ-BALlESTA, M.C.; DOMINGUEZ-PERLES, R.; MORENO, D.A.; MURIES, B.; ALCARAZ-LÓPEZ, C.; BASTÍAS, E.; GARCÍA-VIGUERA, C.; CARVAJAL, M. Minerals in Plant Food: Effect of Agricultural Practices and Role in Human Health. Agronomy for Sustainable Development, v.30, n. 1, 2010.

MASAMBA, K.G.; NGUYEN, M. Determination and com-parison of vitamin C, calcium and potassium in four selected conventionally and organically grown fruits and vegetables. African Journal of Biotechnology, v. 7, n. 16, p. 2915-2919, 2008.

MERTEN, G.H.; MINELLA, J.P. Qualidade da água em bacias hidrográficas rurais: um desafio atual para a sobrevivência futura. Agroecologia e Desenvolvimento Rural Sustentável, v. 3, n. 4, p.33-38, 2002.

NISHA, P.; SINGHAL, R.; PANDIT, A. A study on the degradation kinetics of visual green colour in spinach (Spinacea oleracea L.) and the effect of salt therein. Journal of Food Engineering, v. 64, p.135-142, 2004

NISHIRA, E.; INOUE, M.; KONDO, K.; TAKAHASHI, K.; NAKATA, N. Spinach yield and nutritional quality affected by controlled soil water matric head. Agricultural water management, v. 51, n.13, p. 217-229, 2001.

REMBIALKOWSKA, E. Health and sensory quality of potatoes and selected vegetables from organic farms. Fund. Roz-wój SGGW Warszawa [in Polish], 2000.

SCHUPHAN, W. Nutritional value of crops as influenced by organic and inorganic fertilizer treatments. Qualitas Plantarum, v. 23, p. 333-358, 1974.

SILVA, H. Determinação do teor de metais pesados em produtos vegetais frescos. Lisboa, 2006. Trabalho de Fim de Curso-monografia (Engenharia Alimentar)- Instituto Superior de Agronomia- Universidade Técnica de Lisboa. Lisboa.

SILVA JUNIOR A.A. Efeitos da adubação mineral e orgânica em repolho. Agropecuária Catarinense, v. 4, p. 53-56, 1991.

SILVA, R.D.P. Determinação do teor de ferro de beterrabas adubadas com dois tratamentos diferenciados: orgânico e convencional. Paraná, 2011. 36 f. Trabalho de conclusão de curso (Curso Superior em Tecnologia de Alimentos) - Universidade Tecnológica Federal do Paraná.

SMITH, B.L. Organic foods vs. supermarket foods: element levels. Journal of Applied Nutrition, v. 45, n. 1, p. 35-39, 1993. 
STOPPELLI, I.M.B.S.; MAGALHÃES, C.P. Saúde e segurança alimentar: a questão dos agrotóxicos. Ciências \& Saúde Coletiva, v. 10, p. 91-100, 2005.

WELCH, R.M.; GRAHAM, R.D. (2004) Breeding for micronutrients in staple food crops from a human nutrition perspective. Journal of Experimental Botany, v. 55, p. 353-364, 2004.

WORTHINGTON, V. Nutritional quality of organic versus conventional fruits, vegetables, and grains. Journal of Alternative and Complementary Medicine, v. 7, p. 161-173, 2001. 